Acta Physica Academiae Scientiarum Hungaricae, Tomus 24 (4), pp. $427-429$ (1968)

\title{
DER FEHLER DER NÄHERUNGSWELLENFUNKTION VON QUANTENCHEMISCHEN SYSTEMEN
}

\author{
Von \\ Szidónia Barát \\ PHYSIKALISCHES INSTITUT DER UNIVERSITÄT FÜR TECHNISCHE WISSENSCHAFTEN, BUDAPEST
}

(Eingegangen: 6. VI. 1967)

In der Quantenchemie bedeutet die Bestimmung der Eigenschaften von Atomen und Molekülen die Lösung der Schrödinger'schen Gleichung des untersuchten Systems.

Im allgemeinen ist

$$
(\boldsymbol{H}-\boldsymbol{E}) \psi=\mathbf{0} \text {, wobei }\left\langle\psi^{*} \mid \psi\right\rangle=1 .
$$

Abgesehen von einigen einfachen Fällen, kennen wir die exakte Lösung der Eigenwert-Aufgabe (1) nicht. Deswegen wurden Näherungsverfahren ausgearbeitet, mit Hilfe deren die die Gleichung (1) befriedigende Wellenfunktion und der dazu gehörende Eigenwert annähernd bestimmt werden können. Kann auch die Genauigkeit der Näherung angegeben werden, so ist die aus dem Endresultat abgeleitete Folgerung mit voller Gewissheit abfassbar.

In den meisten Fällen wird eine Wellenfunktion auf Grund des Energieminimumprinzips gelöst. Die Näherungswellenfunktion $\varphi$ wird derart variiert, dass der zu erwartende Wert der Energie ein Minimum ergibt:

$$
\langle\varphi|H| \varphi\rangle=\langle H\rangle=\min , \text { wobei }\langle\varphi \mid \varphi\rangle=1 .
$$

Die so abgeleitete Energie nähert sich in vielen Fällen der Energie des Grundzustandes mit befriedigender Genauigkeit. Das gute Ergebnis bezüglich der Energie ist jedoch keineswegs der Masstab der Güte der Näherungswellenfunktion. Die Genauigkeit des Erwartungswertes eines Operators wird von einer Berechnung mit der Näherungsfunktion, die durch die Energievariationsmethode bestimmt wurde, noch nicht sichergestellt.

Man soll hier zwischen zwei grundverschiedenen Verfahren unterscheiden:

a) entweder vergleicht man die Näherungswellenfunktion mit der exakten Wellenfunktion und bestimmt in irgendeiner Weise den Fehler der Näherung, und gibt den Fehler an:

$$
\int(\psi-\varphi)^{2} d \tau=\eta^{2}
$$


b) oder führt spezielle Untersuchungen zur Bestimmung der Güte der Näherungswellenfunktion aus und schlägt Mittel und Wege zu einer besseren Näherung vor.

Wir werden uns hier nur mit der in Punkt a) umschriebenen Fehlerschätzung befassen.

Im Zusammenhang mit der Fehlerschätzung der Näherungsberechnungen geben die Autoren die obere und die untere Grenze des Fehlers der Energie an. Mehrere Autoren befassen sich mit der oberen Grenze des Fehlers der Näherungswellenfunktion. Wir versuchen nun die untere Grenze dieses Fehlers folgenderweise zu bestimmen:

Seie irgendeine Näherung der exakten Wellenfunktion $\psi$ die reelle und normierte Funktion $\varphi$. Dann gilt

$$
\psi=\varphi+A
$$

wobei $\triangle$ dem Fehler der Näherung entspricht.

Der Erwartungswert des Hermitischen Operators $f$ ist

$$
\begin{aligned}
\langle\varphi|f| \psi\rangle & =\langle\varphi|f| \varphi\rangle+2\langle\Delta|f| \varphi\rangle+\langle\Delta|f| \Delta\rangle= \\
& =\langle\varphi|f| \varphi\rangle+\langle\Delta|f| \varphi\rangle+\Delta|f| \psi-\Delta\rangle+\langle\Delta|f| \Delta\rangle= \\
& =\langle\varphi|f| \varphi\rangle+\langle\Delta|f| \varphi\rangle+\langle\Delta|f| \psi\rangle
\end{aligned}
$$

somit gilt

$$
\langle\psi|f| \psi\rangle-\langle\varphi|f| \varphi\rangle=\langle\Delta|f| \varphi\rangle+\langle\Delta|f| \psi\rangle
$$

Auf Grund der Schwarz'schen Gleichung kann geschrieben werder.

$$
\begin{aligned}
& \langle\Delta|f| \varphi\rangle \leq\left[\langle\Delta \mid \Delta\rangle\left\langle\varphi\left|f^{2}\right| \varphi\right\rangle\right]^{1 / 2}, \\
& \langle\Delta|f| \psi\rangle \leq\left[\langle\Delta \mid \Delta\rangle\left\langle\varphi\left|f^{2}\right| \psi\right\rangle\right]^{1 / 2} .
\end{aligned}
$$

Aus (5) und (6) ergibt sich, dass die linke Seite von (4) in der Form

$$
\langle\psi|f| \psi\rangle-\langle\varphi|f| \varphi\rangle \leq[\langle\Delta \mid \mathcal{U}\rangle]^{1 / 2}\left\{\left[\left\langle\varphi\left|f^{2}\right| \varphi\right\rangle\right]^{1 / 2} \dashv\left[\left\langle\psi\left|f^{2}\right| \psi\right\rangle\right]^{1 / 2}\right\}
$$

geschrieben werden kann.

Quadratwurzel des Integrals des Fehlerquadrats:

$$
\langle\Delta \mid \Delta\rangle^{1 / 2} \geq \frac{\langle\psi|f| \psi\rangle-\langle\varphi|f| \varphi\rangle}{\left\langle\psi\left|f^{2}\right| \psi\right\rangle^{1 / 2}+\left\langle\varphi\left|f^{2}\right| \varphi\right\rangle^{1 / 2}} .
$$

Falls für (2) angenommen wird, dass

$$
\psi=\varphi-\mathcal{A}
$$


dann gilt

$$
\begin{aligned}
\langle\varphi|f| \psi\rangle & =\langle\varphi|f| \varphi\rangle-2\langle\Delta|f| \varphi\rangle+\langle\Delta|f| \Delta\rangle= \\
& =\langle\varphi|f| \varphi\rangle-\langle\Delta|f| \varphi\rangle-\langle\Delta|f| \psi\rangle
\end{aligned}
$$

Mit Hinsichı auf (5) und (6) ist

$$
\langle\varphi|f| \varphi\rangle-\langle\psi|f| \psi\rangle \leq\langle A \mid \Delta\rangle^{1 / 2}\left\{\left\langle\varphi\left|f^{2}\right| \varphi\right\rangle^{1 / 2}+\left\langle\psi\left|f^{2}\right| \psi\right\rangle^{1 / 2}\right\}
$$

woraus

$$
\langle\Delta \mid \Delta\rangle^{1 / 2} \geq \frac{\langle\varphi|f| \varphi\rangle-\langle\varphi|f| \psi\rangle}{\left\langle\psi\left|f^{2}\right| \psi\right\rangle^{1 / 2}+\left\langle\varphi\left|f^{2}\right| \varphi\right\rangle^{1 / 2}} .
$$

Die rechten Seiten von (8) und (8a) unterscheiden sich nur im Vorzeichen, eines von beiden ist also immer negativ. Weil das sich an der linken Seite der Gleichung befindende Fehlerquadrat-Integral nur positiv sein kann, ist eine Ungleichheit, wo sich auf der rechten Seite eine negative Quantität befindet, nichtssagend. Unter Ausklammerung dieses Falles gilt:

$$
\langle\Delta \mid \Delta\rangle^{1 / 2} \geq \frac{|\langle\psi|f| \psi\rangle-\langle\varphi|f| \varphi\rangle|}{\left\langle\psi\left|f^{2}\right| \psi\right\rangle^{1 / 2}+\left\langle\varphi\left|f^{2}\right| \varphi\right\rangle^{1 / 2}}
$$

Für die untere Grenze der Näherungsfunktion erhielten wir dadurch eine Formel, in der nur messbare und berechenbare Quantitäten vorkommen.

\section{LITERATUR}

H. Preuss, Z. Naturforschg. 16a, 698, 1961. 